\title{
Successful Conservative Treatment of Cardiac Rupture Associated with Takotsubo Syndrome
}

\author{
Yoshinori Mano, Akiyasu Baba, Hiroaki Sukegawa, Mitsuaki Sawano, \\ Takafumi Nishiyama and Takahiro Ohki
}

\begin{abstract}
:
We herein report a 75-year-old woman who was diagnosed with Takotsubo syndrome (TTS) complicated by left ventricular outflow tract obstruction on admission. Treatment with beta-blocker and anticoagulant was started; however, her hemoglobin level decreased gradually, and computed tomography performed one week later revealed hemopericardium. Oozing-type cardiac rupture was suspected; therefore, we discontinued heparin treatment. Finally, she recovered uneventfully without cardiac surgery. It is noteworthy that cardiac rupture may occur with TTS, especially in patients treated with prophylactic anticoagulation therapy for apical thrombus. Furthermore, conservative, careful observation is an alternative approach in patients with oozingtype cardiac rupture associated with TTS.
\end{abstract}

Key words: takotsubo syndrome, cardiac rupture, hemopericardium, apical thrombus, prophylactic anticoagulation therapy, left ventricular outflow tract obstruction

(Intern Med 60: 2097-2102, 2021)

(DOI: 10.2169/internalmedicine.5831-20)

\section{Introduction}

Takotsubo syndrome (TTS) is typically characterized by transient regional wall motion abnormality in the apical segments of the left ventricle, extension beyond a single coronary territory, and the absence of obstructive coronary artery disease (1-3). TTS has been generally recognized as a benign disease if patients have no severe underlying disease (1-3). However, recent studies have suggested that TTS may be associated with serious complications and that its prevalence is probably underestimated (4-6). It has been shown that approximately $20 \%$ of TTS patients have serious in-hospital complications (7) and that the morbidity and mortality rates of TTS are comparable to those of acute coronary syndrome $(6,7)$.

We herein report a case of TTS complicated by cardiac rupture successfully treated without surgery.

\section{Case Report}

A 75-year-old woman was admitted to our hospital for chest pain and dyspnea that began the previous day. On admission, her blood pressure was $107 / 63 \mathrm{mmHg}$, and her pulse rate was 69 beats per minute. An electrocardiogram showed sinus rhythm, abnormal Q wave in leads V3-6, slight ST-segment elevation in leads V3-5, and inverted T waves in leads V2-5 (Fig. 1). Her laboratory results showed increased cardiac troponin I and brain natriuretic peptide levels (Table 1). An echocardiogram revealed akinesis in the left ventricular (LV) apical segments and hyperkinesis in the LV basal segments with an LV ejection fraction of 52\% (Fig. 2A, B). Significant LV outflow tract obstruction (LVOTO) with a gradient of $38 \mathrm{mmHg}$ accompanied by moderate mitral regurgitation was also observed (Fig. 2C, D).

To differentiate TTS from acute coronary syndrome, we performed urgent cardiac catheterization. Coronary angiography did not reveal any coronary artery stenosis (Fig. 3A, B), and left ventriculography demonstrated apical ballooning and basal hyperkinesis (Fig. 3C, D). The patient was diagnosed with TTS and received beta-blocker (carvedilol $5 \mathrm{mg}$ daily) and anticoagulation therapy with heparin (continuous infusion of 500-600 units per hour) to prevent apical throm-

Department of Cardiology, Tokyo Dental College, Ichikawa General Hospital, Japan

Received: July 10, 2020; Accepted: December 10, 2020; Advance Publication by J-STAGE: February 8, 2021

Correspondence to Dr. Yoshinori Mano, yoshinori.mano@gmail.com 


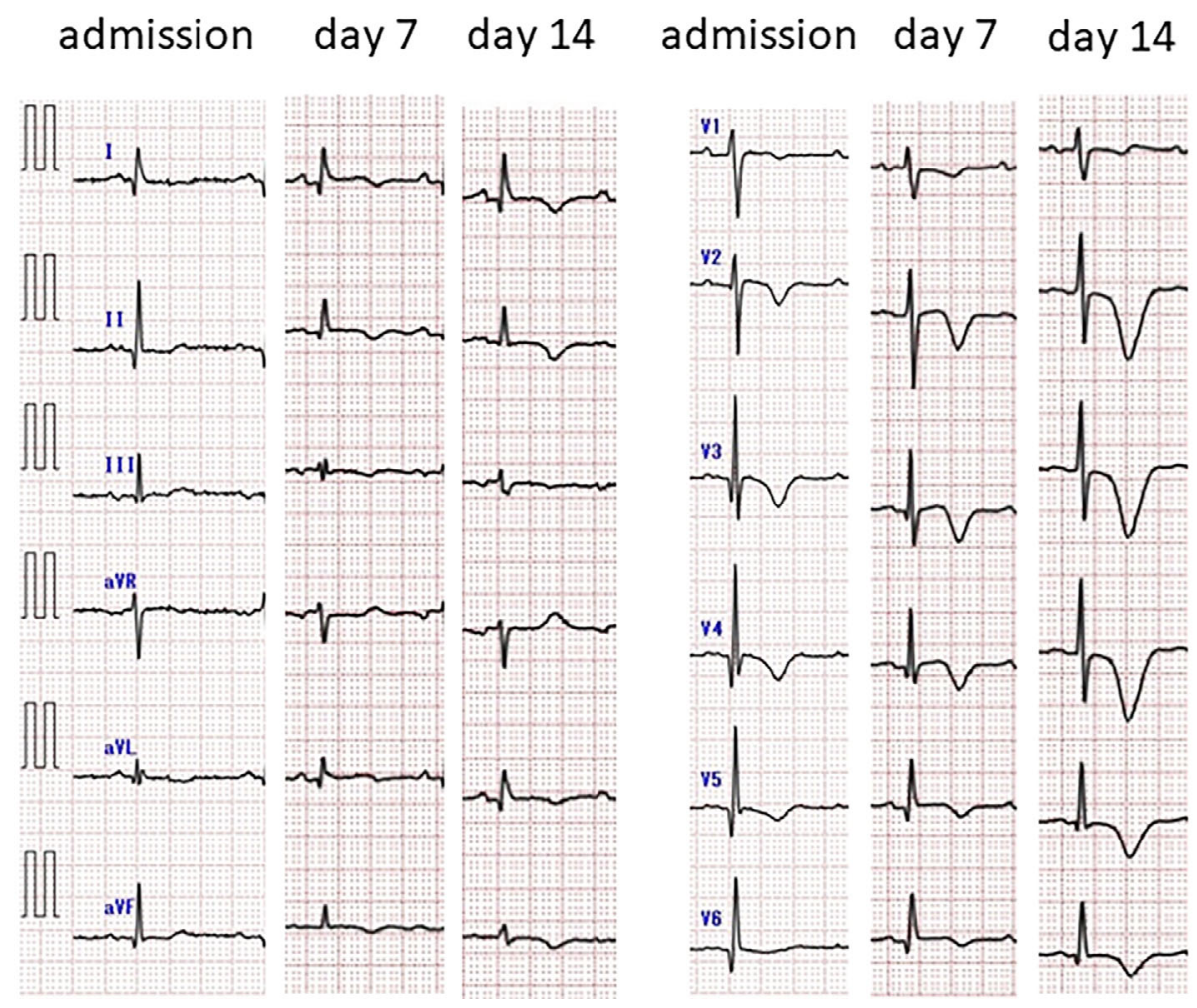

Figure 1. The time-course of 12-lead electrocardiography; The electrocardiogram on admission showed abnormal $Q$ wave in leads V3-6, slight ST-segment elevation in leads V3-5 and inverted T waves in leads V2-5. On day 7, persistent ST-segments elevation was observed in leads V3-6. On day 14, a deeply inverted T-wave was observed in leads V2-6.

Table 1. Laboratory Data on Admission.

\begin{tabular}{lclc}
\hline WBC $(/ \mu \mathrm{L})$ & 6,200 & TP $(\mathrm{g} / \mathrm{dL})$ & 6.2 \\
RBC $(/ \mu \mathrm{L})$ & $390 \times 10^{4}$ & TB $(\mathrm{mg} / \mathrm{dL})$ & 0.6 \\
$\mathrm{Hb}(\mathrm{g} / \mathrm{dL})$ & 12.1 & AST $(\mathrm{U} / \mathrm{L})$ & 38 \\
$\mathrm{Ht}(\%)$ & 35.3 & ALT $(\mathrm{U} / \mathrm{L})$ & 25 \\
Platelet $(/ \mu \mathrm{L})$ & $15.6 \times 10^{4}$ & $\mathrm{LDH}(\mathrm{U} / \mathrm{L})$ & 193 \\
& & ALP $(\mathrm{U} / \mathrm{L})$ & 216 \\
LDL-C $(\mathrm{mg} / \mathrm{dL})$ & 128 & $\gamma-\mathrm{GTP}(\mathrm{U} / \mathrm{L})$ & 24 \\
HDL-C $(\mathrm{mg} / \mathrm{dL})$ & 60 & $\mathrm{UN}(\mathrm{mg} / \mathrm{dL})$ & 12.4 \\
TG $(\mathrm{mg} / \mathrm{dL})$ & 183 & Creatinine $(\mathrm{mg} / \mathrm{dL})$ & 0.56 \\
Glucose $(\mathrm{mg} / \mathrm{dL})$ & 102 & $\mathrm{Uric}$ acid $(\mathrm{mg} / \mathrm{dL})$ & 3.4 \\
HbA1c $(\%)$ & 5.8 & Na $(\mathrm{mEq} / \mathrm{L})$ & 140 \\
& & $\mathrm{~K}(\mathrm{mEq} / \mathrm{L})$ & 3.8 \\
CK $(\mathrm{U} / \mathrm{L})$ & 147 & $\mathrm{Cl}(\mathrm{mEq} / \mathrm{L})$ & 106 \\
Trop I $(\mathrm{ng} / \mathrm{mL})$ & 2.26 & $\mathrm{CRP}(\mathrm{mg} / \mathrm{dL})$ & 0.14 \\
BNP $(\mathrm{pg} / \mathrm{mL})$ & 688 & & \\
\hline
\end{tabular}

WBC: white blood cell, RBC: red blood cell, Hb: hemoglobin, Ht: hematocrit, LDL-C: low density lipoprotein cholesterol, HDL-C: high density lipoprotein cholesterol, TG: triglyceride, HbAlc: hemoglobin Alc, CK: creatine kinase, Trop I: troponin I, BNP: brain natriuretic peptide, TP: total protein, TB: total bilirubin, AST: aspartate aminotransferase, ALT: alanine transaminase, LDH: lactate dehydrogenase, ALP: alkaline phosphatase, $\gamma$-GTP: $\gamma$-glutamyl transpeptidase, UN: urea nitrogen, CRP: Creactive protein

bosis, with a target activated partial thromboplastin time (APTT) of 1.5 times the control value (45-60 seconds).

After the initiation of beta-blocker, her chest discomfort gradually decreased; however, laboratory results showed a gradual decrease in her hemoglobin levels and an elevation in her liver enzymes (Table 2). On the fifth day of hospitalization, we reduced the dosage of heparin to $400 \mathrm{units} / \mathrm{h}$, although we could not identify the cause of her anemia. At this point, her blood pressure was $103 / 66 \mathrm{mmHg}$, pulse rate was 84 beats per minute, and APTT was 50.2 seconds. On the seventh day, we performed plain and contrast-enhanced computed tomography (CT) and found a moderate amount of pericardial effusion, measuring $15 \mathrm{~mm}$ in thickness, with high CT attenuation values (ranging from 35 to 48 Hounsfield units), which was suggestive of hemopericardium (Fig. 4A), accompanied by hepatic congestion and pleural effusion. No abnormality or bleeding source was observed in other organs. Her echocardiogram also showed a moderate amount of pericardial effusion; however, the apical ballooning had virtually resolved (Fig. 4B, C). Furthermore, the LVOT peak pressure gradient decreased to $17 \mathrm{mmHg}$, and mitral regurgitation improved to a mild degree. At this point, her blood pressure was $106 / 63 \mathrm{mmHg}$, her pulse rate was 89 beats per minute, and her APTT level had reached the subtherapeutic level of 38.9 seconds. Her electrocardiogram indicated persistent subtle elevation of the ST-segment in leads V3-6 (Fig. 1). Given this clinical course and findings, the presence of oozing-type cardiac rupture was suspected.

We discontinued heparin treatment; however, the patient was followed closely without surgical treatment. Her condition improved, and the pericardial effusion decreased gradu- 

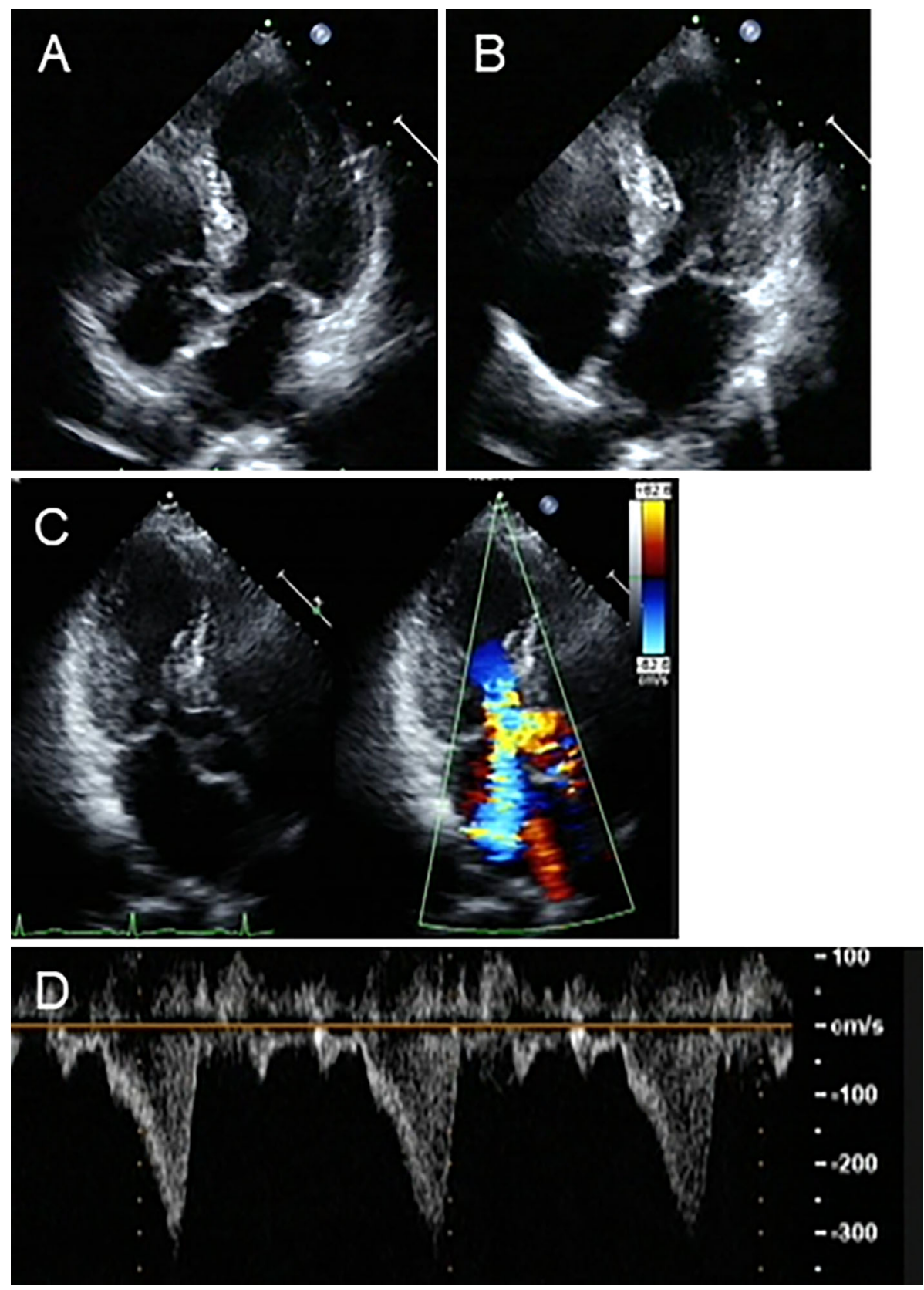

Figure 2. Echocardiographic images following admission. The echocardiogram during diastole (A) and systole (B) showed apical akinesis, with no pericardial effusion. LV outflow tract obstruction (LVOTO) accompanied by moderate mitral regurgitation was observed (C). Doppler waveforms indicated that the peak velocity of the left ventricular outflow obstruction was $3.1 \mathrm{~m} / \mathrm{s}$ (D).

ally (Fig. 4D, E). Ultimately, she recovered uneventfully without surgery and was discharged home. Cardiac magnetic resonance $(\mathrm{CMR})$ imaging performed six months after discharge showed no regional wall motion abnormality, pericardial effusion, or late gadolinium enhancement (LGE) (Fig. 4F), which was consistent with the diagnosis of TTS.

\section{Discussion}

This is the first known report of a case of TTS complicated by cardiac rupture that was successfully treated without surgery. TTS has been generally regarded as a benign disease with complete recovery of the LV function. How- ever, it is now considered to be associated with severe clinical complications, including heart failure, cardiogenic shock due to LV outflow tract obstruction, apical thrombus, ventricular arrhythmias, and cardiac rupture (1-5).

Significant LVOTO has been observed in $10-25 \%$ of patients with TTS $(4,6)$. It is often accompanied by mitral regurgitation due to systolic anterior motion of the mitral leaflet and leaflet tethering by displacement of the subvalvular mitral valve apparatus. LVOTO and mitral regurgitation are sometimes associated with severe conditions, including cardiogenic shock. Therefore, evaluating these entities by echocardiography is important in patients with TTS. In the presence of LVOTO, the use of inotropic agents or nitroglycerin, 


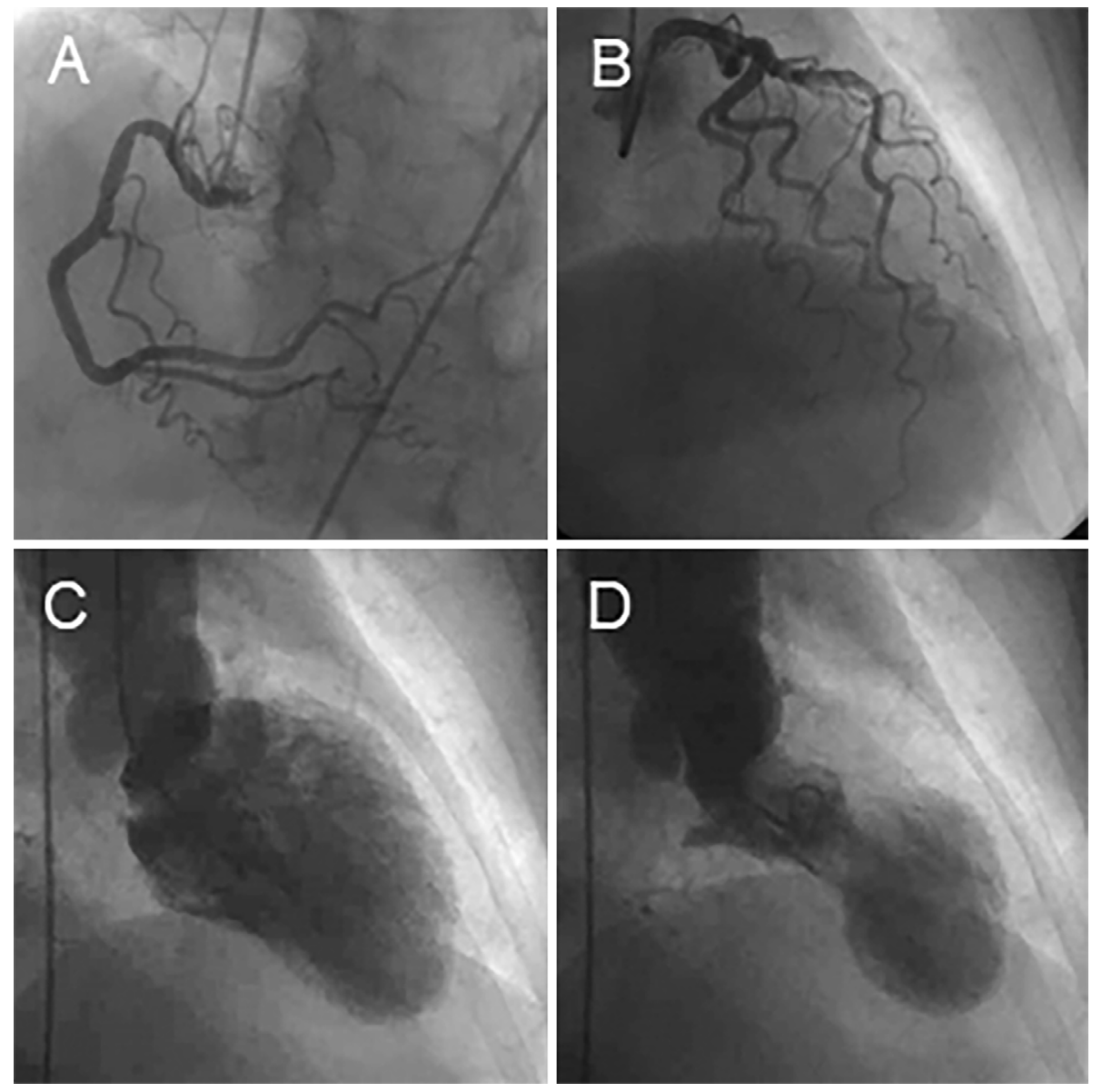

Figure 3. Angiographic images following admission; A coronary angiogram demonstrated no obstructive coronary artery disease in the right (A) or left (B) coronary systems. A left ventriculogram, during diastole (C) and systole (D), indicated apical ballooning and basal hyperkinesis consistent with Takotsubo syndrome.

which may exacerbate LVOTO, should be avoided, and treatment with a beta-blocker should instead be considered (4). In our case, we used a beta-blocker, and LVOTO had resolved by the seventh day following admission.

LV apical thrombus is found in $2-8 \%$ of TTS patients (4). Apical thrombus has been suggested as a potential source of emboli, such as cerebral ischemic stroke $(1-4,8,9)$. Therefore, anticoagulation therapy is recommended if LV thrombus is detected (4). In contrast, for TTS patients without LV thrombus, the role of prophylactic anticoagulation therapy remains to be determined. Although evidence is lacking, in TTS patients with severe LV dysfunction and a low bleeding risk, anticoagulation therapy to prevent LV thrombus may be appropriate until the resolution of regional wall motion abnormality is confirmed via cardiac imaging $(2-4,6,10)$. However, such therapy can be detrimental if cardiac rupture occurs.

Of note, a small amount of non-hemorrhagic inflammatory effusion complicated with TTS has been reported previously $(11,12)$. However, in our case, a moderate amount of pericardial effusion with high CT attenuation values suggestive of hemorrhagic pericardial effusion $(13,14)$, accompanied by the progression of anemia, was observed. Thus, the patient was diagnosed with cardiac rupture associated with TTS, although the rupture site could not be definitively detected.

Cardiac rupture is a rare but life-threatening complication of TTS unless recognized expeditiously and repaired surgically $(3,15)$. It may occur two to eight days after the symptom onset (4). The risk factors for cardiac rupture complicating TTS are female sex, old age, high blood pressure, persistent ST segment elevation, and the absence of betablocker therapy $(4,10,15,16)$. LVOTO may also be associated with cardiac rupture (4). Our patient was an elderly woman; persistent ST-segment elevation and LVOTO were observed. One report found that 10 out of 12 patients (83\%) with cardiac rupture died (15). In our case, we discontinued heparin therapy; however, no surgical intervention was performed because the patient was not hemodynamically compromised, and her regional wall motion abnormality and the degree of LVOTO had substantially improved. Deciding whether to perform surgery or conservatively follow the hemodynamics is very difficult. There are cases that require surgical intervention to save patients' lives. However, elderly patients accompanied by multiple comorbidities tend to have a high rate of complications during and after cardiac sur- 
Table 2. Time Course of Hemoglobin, D-dimer, CRP and Liver Enzymes.

\begin{tabular}{lccccccc}
\hline & $\begin{array}{c}\text { Day 1 } \\
\text { (on admission) }\end{array}$ & Day 2 & Day 5 & Day 7 & Day 9 & Day 25 & Day 40 \\
\hline Hb (g/dL) & 12.1 & 11.2 & 9.9 & 8.6 & 8.4 & 9.2 & 11.3 \\
D-dimer $(\mu \mathrm{g} / \mathrm{mL})$ & - & - & 1.3 & - & 8.7 & 13.1 & 1.5 \\
CRP $(\mathrm{mg} / \mathrm{dL})$ & 0.14 & 0.32 & 6.27 & 8.36 & 5.58 & 0.68 & 0.29 \\
& & & & & & & \\
AST (U/L) & 38 & 30 & 68 & 199 & 70 & 30 & 18 \\
ALT (U/L) & 25 & 19 & 62 & 237 & 155 & 52 & 14 \\
ALP (U/L) & 216 & 184 & 299 & 977 & 977 & 614 & 355 \\
$\gamma$-GTP (U/L) & 24 & 21 & 75 & 301 & 298 & 164 & 84 \\
\hline
\end{tabular}

Abbreviations as in Table 1.
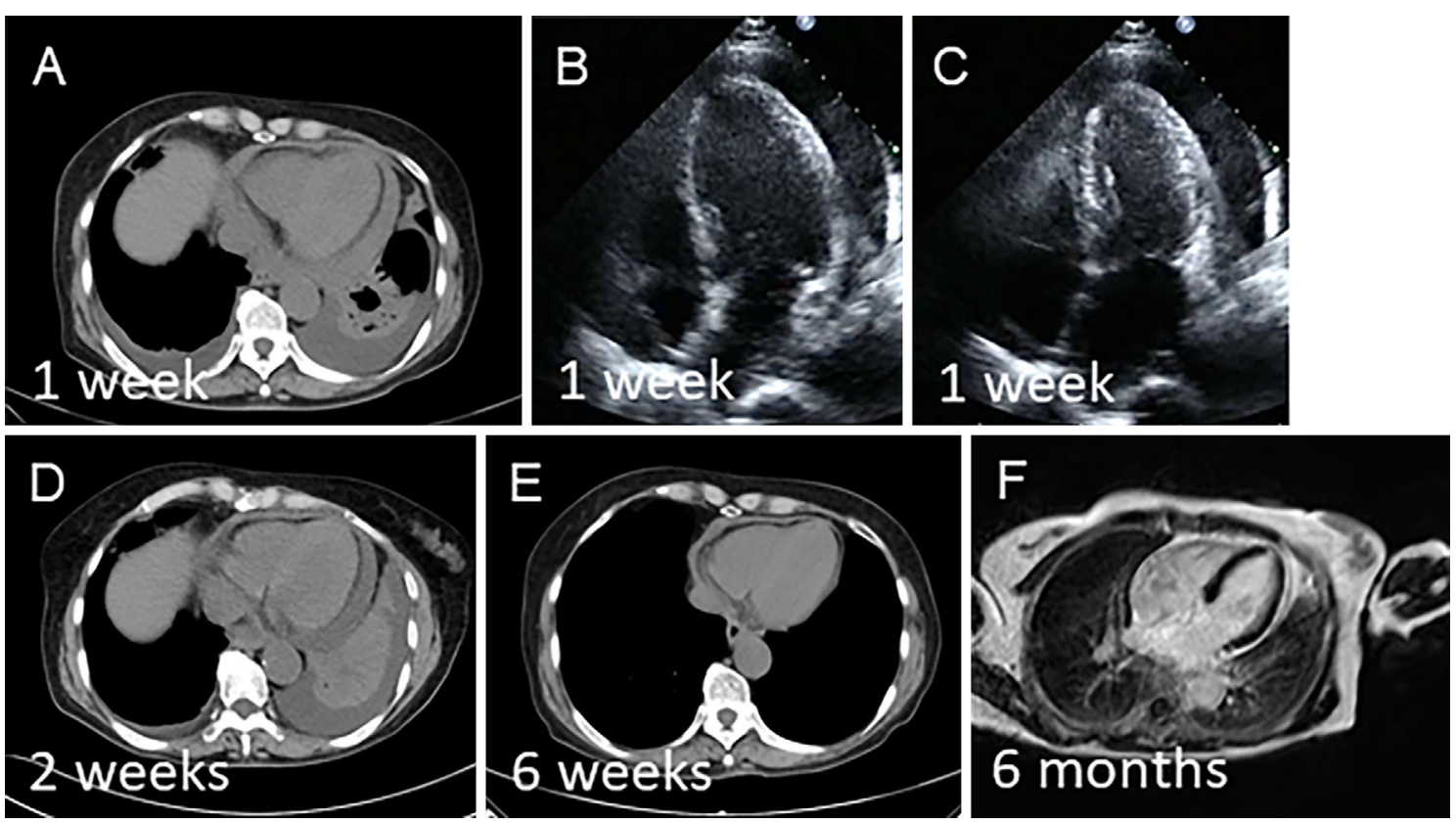

Figure 4. Computed tomography (CT) performed on the seventh day following admission showed a moderate amount of pericardial effusion with high $\mathrm{CT}$ attenuation values suggestive of hemopericardium (A). Echocardiography also showed pericardial effusion, with improved left ventricular segmental asynergy during diastole (B) and systole (C). CT performed two weeks (D) and six weeks (E) later showed that the pericardial effusion had decreased gradually. Cardiac magnetic resonance imaging (F) performed six months later showed no wall motion abnormalities, pericardial effusion, or late gadolinium enhancement, which is consistent with the progress of Takotsubo syndrome.

gery.

One limitation of our case report was that we could not confirm the rupture site of LV. In the past decade, CMR has emerged as a key diagnostic tool for the assessment of TTS (4, 17-19). CMR can provide information on myocardial tissue characteristics in addition to the accurate visualization of wall motion abnormalities $(6,17,18)$. Typically, the absence of LGE on CMR, which reflects myocardial damage, is considered an important finding differentiating TTS from myocardial infarction $(4,17,18)$. However, recent studies have revealed that low-intensity LGE may be present in some patients with TTS during the acute phase but absent at follow-up CMR (4, 17-19). The mechanisms and clinical implications of LGE in the acute phase have not been fully elucidated; however, it may be associated with the severity, increased serum troponin levels, and a prolonged recovery to normal clinical findings in TTS patients (17-19). In our case, CMR performed six months after discharge did not show any abnormalities, including LGE. However, had we performed CMR during the acute phase, we would have been able to identify some findings suggestive of cardiac damage leading to cardiac rupture.

\section{Conclusion}

It has been shown that the prognosis of TTS is not as favorable as previously thought. We should keep in mind that cardiac rupture may occur in TTS patients, especially in those treated with prophylactic anticoagulation therapy for 
apical thrombus. Persistent ST-segment elevation and LVOTO may be associated with cardiac rupture. Furthermore, although an individualized risk-benefit profile must be carefully constructed by clinicians, conservative careful observation is a viable alternative in patients with oozing-type cardiac rupture associated with TTS.

The authors state that they have no Conflict of Interest (COI).

\section{References}

1. Hurst RT, Prasad A, Askew JW 3rd, Sengupta PP, Tajik AJ. Takotsubo cardiomyopathy: a unique cardiomyopathy with variable ventricular morphology. JACC Cardiovasc Imaging 3: 641-649, 2010.

2. Yoshikawa T. Takotsubo cardiomyopathy, a new concept of cardiomyopathy: clinical features and pathophysiology. Int J Cardiol 182: 297-303, 2015.

3. Kurisu S, Kihara Y. Clinical management of takotsubo cardiomyopathy. Circ J 78: 1559-1566, 2014.

4. Lyon AR, Bossone E, Schneider B, et al. Current state of knowledge on Takotsubo syndrome: a Position Statement from the Taskforce on Takotsubo Syndrome of the Heart Failure Association of the European Society of Cardiology. Eur J Heart Fail 18: 8-27, 2016.

5. Ghadri JR, Wittstein IS, Prasad A, et al. International Expert Consensus Document on Takotsubo Syndrome (Part I): Clinical Characteristics, Diagnostic Criteria, and Pathophysiology. Eur Heart J 39: 2032-2046, 2018.

6. Ghadri JR, Wittstein IS, Prasad A, et al. International Expert Consensus Document on Takotsubo Syndrome (Part II): Diagnostic Workup, Outcome, and Management. Eur Heart J 39: 2047-2062, 2018.

7. Templin C, Ghadri JR, Diekmann J, et al. Clinical features and outcomes of takotsubo (stress) cardiomyopathy. N Engl J Med 373: 929-938, 2015.

8. de Gregorio C, Grimaldi P, Lentini C. Left ventricular thrombus formation and cardioembolic complications in patients with takotsubo-like syndrome: a systematic review. Int J Cardiol 131:
18-24, 2008.

9. Kurisu S, Inoue I, Kawagoe T, et al. Incidence and treatment of left ventricular apical thrombosis in tako-tsubo cardiomyopathy. Int J Cardiol 146: e58-e60, 2011.

10. Kurisu S, Kihara Y. Diagnosis and management of takotsubo cardiomyopathy. Intern Med 54: 1-2, 2015.

11. Eitel I, Lücke C, Grothoff M, et al. Inflammation in takotsubo cardiomyopathy: insights from cardiovascular magnetic resonance imaging. Eur Radiol 20: 422-431, 2010.

12. Nagamori $Y$, Hamaoka T, Murai H, et al. Takotsubo cardiomyopathy complicated by cardiac tamponade due to non-hemorrhagic pericardial effusion: a case report. BMC Cardiovasc Disord 20: 67, 2020.

13. Imazio M, Adler Y. Management of pericardial effusion. Eur Heart J 34: 1186-1197, 2013.

14. Çetin MS, Özcan Çetin EH, Özdemir M, et al. Effectiveness of computed tomography attenuation values in characterization of pericardial effusion. Anatol J Cardiol 17: 322-327, 2017.

15. Kumar S, Kaushik S, Nautiyal A, et al. Cardiac rupture in takotsubo cardiomyopathy: a systematic review. Clin Cardiol 34: 672676, 2011.

16. Tsuji M, Isogai T, Okabe $Y$, et al. Ventricular septal perforation: a rare but life-threatening complication associated with takotsubo syndrome. Intern Med 57: 1605-1609, 2018.

17. Plácido R, Cunha Lopes B, Almeida AG, Rochitte CE. The role of cardiovascular magnetic resonance in takotsubo syndrome. J Cardiovasc Magn Reson 18: 68, 2016.

18. Gaikwad N, Butler T, Maxwell R, et al. Late gadolinium enhancement does occur in Tako-tsubo cardiomyopathy - A quantitative cardiac magnetic resonance and speckle tracking strain study. Int J Cardiol Heart Vasc 12: 68-74, 2016.

19. Naruse $Y$, Sato A, Kasahara $K$, et al. The clinical impact of late gadolinium enhancement in Takotsubo cardiomyopathy: serial analysis of cardiovascular magnetic resonance images. J Cardiovasc Magn Reson 13: 67, 2011.

The Internal Medicine is an Open Access journal distributed under the Creative Commons Attribution-NonCommercial-NoDerivatives 4.0 International License. To view the details of this license, please visit (https://creativecommons.org/licenses/ by-nc-nd/4.0/).

(C) 2021 The Japanese Society of Internal Medicine Intern Med 60: 2097-2102, 2021 